\title{
Use of platelet-rich plasma and modified nanofat grafting in infected ulcers: Technical refinements to improve regenerative and antimicrobial potential
}

\author{
Francesco Segreto ${ }^{1}$, Giovanni Francesco Marangi ${ }^{1}$, Carolina Nobile ${ }^{2}$, Mario Alessandri-Bonetti ${ }^{1}$, \\ Chiara Gregorj ${ }^{2}$, Vincenzo Cerbone ${ }^{3}$, Marco Gratteri ${ }^{1}$, Erika Caldaria ${ }^{1}$, Maria Cristina Tirindelli ${ }^{2}$, \\ Paolo Persichetti ${ }^{1}$ \\ ${ }^{1}$ Department of Plastic, Reconstructive and Aesthetic Surgery and ${ }^{2}$ Department of Hematology, Stem Cell Transplantation, Transfusion \\ Medicine and Cellular Therapy, Campus Bio-Medico of Rome University, Rome; ${ }^{3}$ Department of Orthopedics and Traumatology, II \\ University Clinic, G. Pini Orthopaedic Institute, Milan, Italy
}

Background Surgical reconstruction of chronic wounds is often infeasible due to infection, comorbidities, or poor viability of local tissues. The aim of this study was to describe the authors' technique for improving the regenerative and antimicrobial potential of a combination of modified nanofat and platelet-rich plasma (PRP) in nonhealing infected wounds.

Methods Fourteen patients met the inclusion criteria. Fat tissue was harvested from the lower abdomen following infiltration of a solution of 1,000 mL of NaCl solution, $225 \mathrm{mg}$ of ropivacaine, and $1 \mathrm{mg}$ of epinephrine. Aspiration was performed using a 3-mm cannula with $1-\mathrm{mm}$ holes. The obtained solution was decanted and mechanically emulsified, but was not filtered. Non-activated leukocyte-rich PRP (naLR-PRP) was added to the solution before injection. Patients underwent three sessions of injection of 8-mL naLR-PRP performed at 2-week intervals.

Results Thirteen of 14 patients completed the follow-up. Complete healing was achieved in seven patients $(53.8 \%)$. Four patients $(30.8 \%)$ showed improvement, with a mean ulcer width reduction of $57.5 \% \pm 13.8 \%$. Clinical improvements in perilesional skin quality were reported in all patients, with reduced erythema, increased thickness, and increased pliability. An overall wound depth reduction of $76.6 \% \pm 40.8 \%$ was found. Pain was fully alleviated in all patients who underwent re-epithelization. A mean pain reduction of $42 \% \pm 33.3 \%$ (as indicated by visual analog scale score) was found in non-re-epithelized patients at a 3-month follow-up.

Conclusions The discussed technique facilitated improvement of both the regenerative and the antimicrobial potential of fat grafting. It proved effective in surgically-untreatable infected chronic wounds unresponsive to conventional therapies.

Keywords Platelet-rich plasma / Anti-infective agents / Tissue grafts / Ulcer / Wounds
Correspondence: Francesco Segreto Department of Plastic, Reconstructive and Aesthetic Surgery, Campus BioMedico of Rome University, Via Alvaro del Portillo 200, 00128 Rome, Italy Tel: $+39-6225418824$ Fax: $+39-6225411936$ E-mail: francescosegreto@gmail.com 


\section{INTRODUCTION}

Chronic wounds are a major issue in modern medicine, with more than 4 million affected patients in the United States alone. Around $\$ 50$ billion USD is spent annually on their treatment [1]. The complications of chronic wounds, which include infection, sepsis, and cancerization, sometimes lead to amputation. The patient's quality of life is reduced by pain and the need for frequent medication, as well as by functional and social limitations. Surgical reconstruction is often challenging, especially in patients with severe comorbidities, local infection, and a lack of local viable tissues. As a consequence, conservative treatment with surgical debridement followed by advanced medications is still a mainstay. However, in some patients, the re-epithelization process is extremely slow or absent due to comorbidities. In such cases, as well as in patients unwilling to undergo surgical intervention, regenerative medicine may be a viable option to speed up re-epithelization, possibly alleviating their symptoms.

Over the last decades, several indications for fat grafting have been described, including volume loss, breast augmentation, and reconstruction. More recently, a worldwide effort was undertaken to investigate the regenerative potential of adipose tissue [2], which extended its use to wound healing, the treatment of radiation damage, and the treatment of peri-anal fistulas, with promising results [3]. Nanofat grafting was originally described in 2013 for the purpose of skin rejuvenation [4]. In the original technique, the fat was mechanically emulsified, leading to fragmentation of mature adipocytes, while retaining some adiposederived stem cells (ADSCs) [4]. Nanofat grafting was subsequently used as an adjuvant therapy for complex wounds [5]. Although the concentration of ADSCs in a nanofat graft is lower than in non-fragmented fat, the nanofat may be injected with thinner needles, improving infiltration in fibro-sclerotic tissues. Moreover, a larger number of tunnels may be established, thus improving the contact surface with the vascularized tissue and promoting ADSC survival and proliferation. The cell fragments resulting from the emulsification process act as pro-inflammatory signals that trigger a regeneration cascade $[4,6]$. However, there is still some reluctance to perform fat grafting of any kind in infected or infection-prone tissues.

Platelet-rich plasma (PRP) has been demonstrated to improve the proliferation and motility of ADSCs $[7,8]$, and several types of platelet derivatives, as well as multiple commercial kits, are currently available [9]. However, conflicting evidence exists in the literature regarding the best PRP product and/or concentration to use to promote ADSC proliferation.

The aims of the present study were to develop a technique to maximize the regenerative and antimicrobial potential of a com- bination of ADSCs and PRP and to assess the efficacy of this combination in the treatment of nonhealing infected wounds. The authors' clinical experience is described.

\section{METHODS}

\section{Patients}

This prospective open-label study was approved by the local institutional review board. Fourteen patients were prospectively enrolled between March 2013 and July 2015 according to the following criteria. The inclusion criteria were a surgical or medical contraindication for major surgery, lack of response to one surgical debridement and advanced medications (polyurethane foam and/or alginate) for at least 3 months, and local infection that failed to respond to systemic antibiotic therapy. The exclusion criterion was life-threatening operatory risk. All patients provided informed consent. Pain reduction was assessed using a visual analog scale (VAS). Patients' demographic characteristics are summarized in Table 1. The mean age of the patients ( \pm standard deviation) was $73.86 \pm 9$ years. The mean initial wound size was $64.9 \pm 111 \mathrm{~cm}^{2}$, while the mean initial wound depth was $8.93 \pm 9.25 \mathrm{~mm}$. The mean initial VAS score was $4.17 \pm 0.75$. Patients were followed up for 12 months (Table 1 ).

\section{Technique}

First, we reviewed the literature for information regarding each step of fat processing (e.g., infiltration) and PRP preparation with the goal of increasing ADSC number, viability, and proliferation. Then, we developed the following technique according to findings from the literature. First, the lower abdomen was infiltrated with a tumescent solution containing $1,000 \mathrm{~mL}$ of $\mathrm{NaCl}$ solution, $225 \mathrm{mg}$ of ropivacaine (AstraZeneca, Basiglio, Italy), and $1 \mathrm{mg}$ of epinephrine (Salf, Cenate Sotto, Italy). A 3-mmwide multiperforated cannula with 1-mm lateral holes was used for fat harvesting. The fat was then decanted for 10 minutes and mechanically emulsified by shifting 30 times between two syringes connected by a $90^{\circ}$ Luer-lock connector. No filtration was performed. A 27-gauge needle was chosen for re-injection. Blood was taken before starting the surgical procedure. Non-activated leukocyte-rich PRP (naLR-PRP) was extracted as previously described [10] and was then added to the modified nanofat graft at a $20 \%$ concentration. The modifications to the conventional nanofat technique [4] are summarized in Table 2.

The treatment protocol was as follows: first, the wound was surgically debrided, and a solution of $80 \%$ nanofat and $20 \%$ naLR-PRP was injected in the wound bed and margins, both intradermally and subcutaneously. Injection was stopped when slight blanching of the tissue was noticed, and a paraffin-embedded 
Table 1. Patients' demographic characteristics

\begin{tabular}{|c|c|c|c|c|c|c|c|c|c|}
\hline $\begin{array}{l}\text { Patient } \\
\text { No. }\end{array}$ & $\begin{array}{l}\text { Age } \\
(\mathrm{yr})\end{array}$ & Sex & Ulcer site & $\begin{array}{l}\text { Ulcer } \\
\text { etiology }\end{array}$ & $\begin{array}{l}\text { Preoperative } \\
\text { wound size } \\
\text { (cm) }\end{array}$ & $\begin{array}{l}\text { Preoperative } \\
\text { wound } \\
\text { depth }(\mathrm{mm})\end{array}$ & $\begin{array}{l}\text { Wound depth } \\
3 \text { months } \\
\text { postoperatively } \\
(\mathrm{mm})\end{array}$ & $\begin{array}{c}\text { Area of re- } \\
\text { epithelization } \\
3 \text { months } \\
\text { postoperatively } \\
(\%)\end{array}$ & Pathogen \\
\hline 1 & 76 & $\mathrm{~F}$ & Inferior limb & Vasculitis & $30 \times 15$ & 7 & 2 & 65 & $\begin{array}{l}\text { Staphylococcus simulans, } \\
\text { Pseudomonas aeruginosa }\end{array}$ \\
\hline 2 & 86 & M & Inferior limb & Vascular & $7 \times 6$ & 4 & 0 & 100 & Proteus mirabilis \\
\hline 3 & 79 & M & Inferior limb & Vascular & $7 \times 4$ & 6 & 0 & 100 & P. mirabilis \\
\hline 4 & 81 & $\mathrm{~F}$ & $\begin{array}{l}\text { Lateral-cervical } \\
\text { region }\end{array}$ & Cobaltotherapy & $5 \times 5$ & 7 & 7 & 0 & $\begin{array}{l}\text { P. aeruginosa, Alcaligenes faecalis, } \\
\text { Corynebacterium striatum }\end{array}$ \\
\hline 5 & 78 & M & Sacral region & Pressure sore & $9 \times 7$ & 25 & 10 & 48 & $\begin{array}{l}\text { Enterococcus faecium, P. mirabilis, } \\
\text { P. aeruginosa }\end{array}$ \\
\hline 6 & 79 & M & Inferior limb & Post-traumatic & $8 \times 4$ & 5 & 0 & 100 & P. mirabilis \\
\hline 7 & 71 & $\mathrm{~F}$ & Inferior limb & Post-traumatic & $6 \times 4$ & 5 & 0 & 100 & $P$. aeruginosa \\
\hline 8 & 68 & $\mathrm{~F}$ & Sacral region & Pressure sore & $7 \times 4$ & 35 & 33 & 0 & $\begin{array}{l}\text { P. mirabilis, Enterococcus faecalis, } \\
\text { Enterococcus avium }\end{array}$ \\
\hline 9 & 75 & $\mathrm{~F}$ & Inferior limb & Post-traumatic & $5 \times 3$ & 3 & 0 & 100 & Staphylococcus aureus (MRSA) \\
\hline 10 & 59 & $\mathrm{~F}$ & Inferior limb & Post-traumatic & $7 \times 6$ & 5 & 0 & 100 & S. aureus (MRSA) \\
\hline 11 & 60 & M & Sacral region & Pressure sore & $8 \times 5$ & 4 & Deceased & Deceased & Staphylococcus epidermis \\
\hline 12 & 78 & $\mathrm{~F}$ & Inferior limb & Vascular & $9 \times 7$ & 8 & 1 & 73 & $\begin{array}{l}\text { P. aeruginosa, S. aureus (MRSA), } \\
\text { Morganella morganii, } \\
\text { Streptococcus agalactiae }\end{array}$ \\
\hline 13 & 60 & $\mathrm{~F}$ & Inferior limb & Vascular & $6 \times 4$ & 4 & 0 & 100 & S. epidermis \\
\hline 14 & 84 & M & Inferior limb & Vascular & $8 \times 4$ & 7 & 2 & 44 & P. aeruginosa, S. simulans \\
\hline
\end{tabular}

\section{Table 2. Modified nanofat grafting technique}

\begin{tabular}{|c|c|}
\hline Step & Technique \\
\hline Infiltration & $\begin{array}{l}\text { 1,000 } \mathrm{mL} \text { of } \mathrm{NaCl} \text { solution, } 225 \mathrm{mg} \text { of ropivacaine, } 1 \mathrm{mg} \text { of } \\
\text { epinephrine }\end{array}$ \\
\hline Donor site & Lower abdomen \\
\hline Aspiration & 3-mm cannula with 1-mm holes connected to a Luer-lock syringe \\
\hline Processing & $\begin{array}{l}\text { Decantation and mechanical emulsification, then addition of naLR- } \\
\text { PRP }\end{array}$ \\
\hline Injection & 27-Gauge syringe \\
\hline
\end{tabular}

gauze was then applied. Two weeks postoperatively, the patients underwent three sessions of 8-mL naLR-PRP injection performed at 2-week intervals. The co-primary endpoints were wound area reduction and wound depth reduction 3 months after surgery.

\section{RESULTS}

Thirteen of the 14 patients completed the follow-up. One patient died from causes unrelated to the surgical procedure. Complete healing was achieved in seven patients (53.8\%) with a mean time to re-epithelization of $9.1 \pm 0.6$ weeks. Four patients (30.8\%) showed improvement, with a mean ulcer width reduction of $57.5 \% \pm 13.8 \%$. Two cases (15.4\%) exhibited no change in ulcer width. A clinical improvement in perilesional skin quality was reported in all patients, with reduced erythema, increased thickness, and increased pliability.

An overall wound depth reduction of $76.6 \% \pm 40.8 \%$ was found (Fig. 1).

Pain was fully alleviated in all patients who underwent re-epithelization. A mean pain reduction (as indicated by VAS score) of $42 \% \pm 33.3 \%$ was observed in non-re-epithelized patients at 3 months postoperatively (Figs. 2, 3).

\section{DISCUSSION}

Chronic wounds are both common and challenging. In patients with such wounds, it is paramount to speed up healing, which may alleviate symptoms. Although both nanofat grafting and PRP have been reported to promote wound healing individually, to date a limited body of evidence exists regarding their use in combination. Combining nanofat and PRP into a single, injectable product adds more technical variables, such as ideal proportions, ideal concentrations, the optimal form of PRP to use, and so on. The technique described herein addresses these variables and describe the outcomes of the combination therapy. In our cohort of nonhealing patients, this technique allowed us to achieve both re-epithelization and pain reduction. Our approach exhibits several advantages over other techniques. Mac- 


\section{Fig. 1. Results at 3-month follow-up}

(A) Percentage of healing and (B) wound depth reduction. ${ }^{\text {al Deceased. }}$
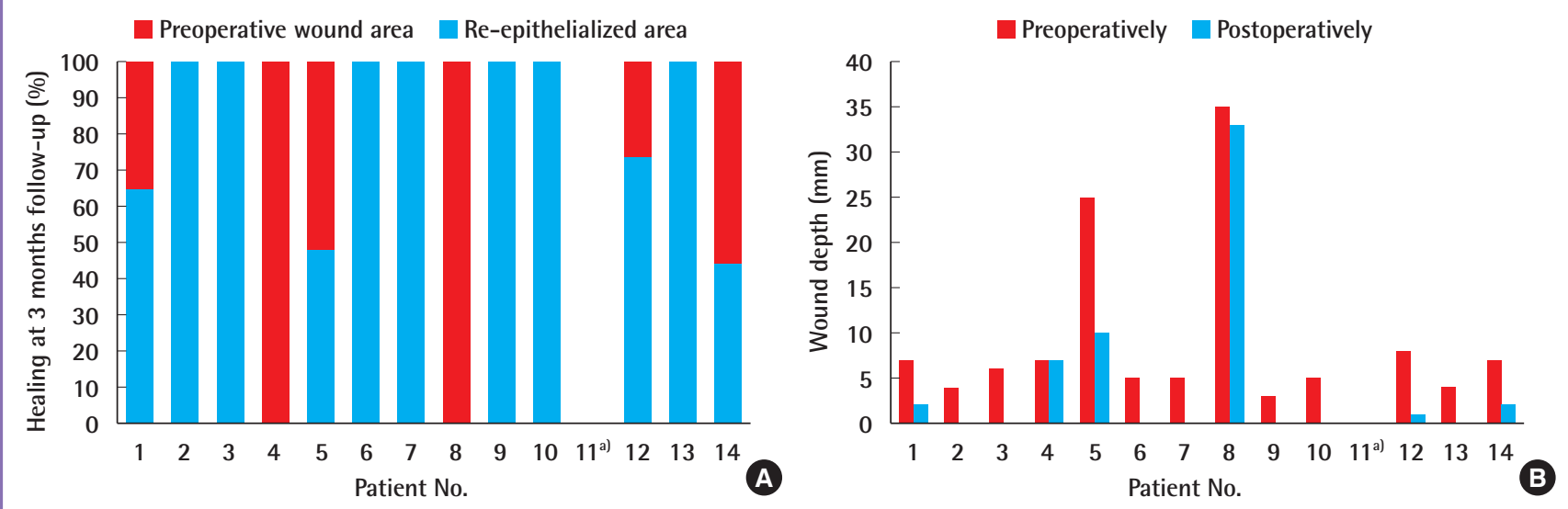

Fig. 2. Infected chronic ulcer (patient No. 6)

(A) Preoperative view. (B) Three-month postoperative view.

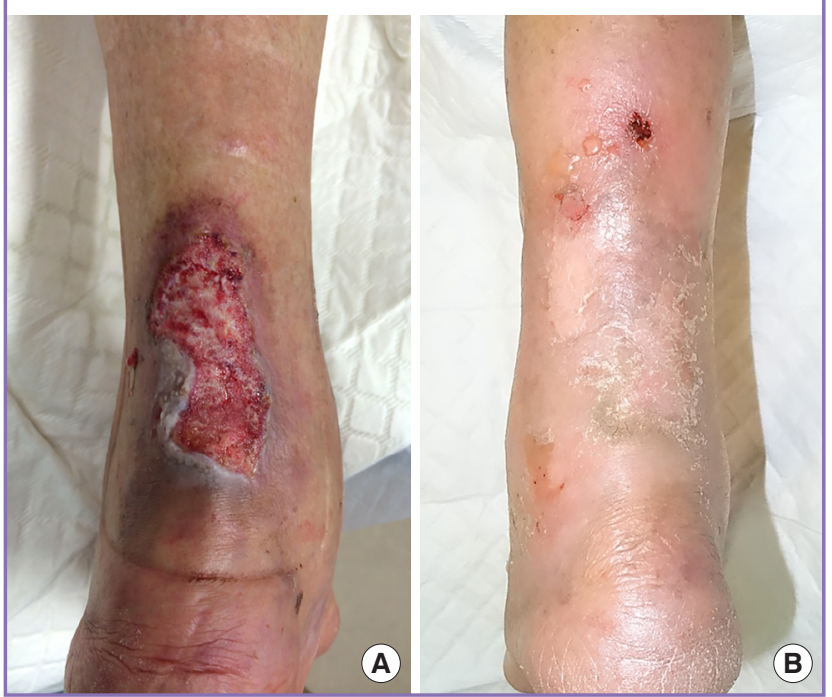

ro-fat grafting has regenerative potential [2] but also has several drawbacks. The cell take in the recipient site is limited by local perfusion and tissue compliance, according to the principle of the graft-to-capacity ratio [11]. Mature adipocytes are less prone to inducing regeneration than the stromal vascular fraction [3]. Moreover, they comprise a large portion of the fat graft and can theoretically "steal" metabolites from the ADSCs to facilitate their own take. As a consequence, reducing the content of mature adipocytes in the injected material may theoretically allow for better survival and differentiation of ADSCs. The nanofat grafting technique [4] allows mature adipocytes to be discarded, preserving the ADSCs. Nevertheless, the ADSC content is lower than in macro-fat grafting. In our approach, harvesting and processing were optimized according to the avail-

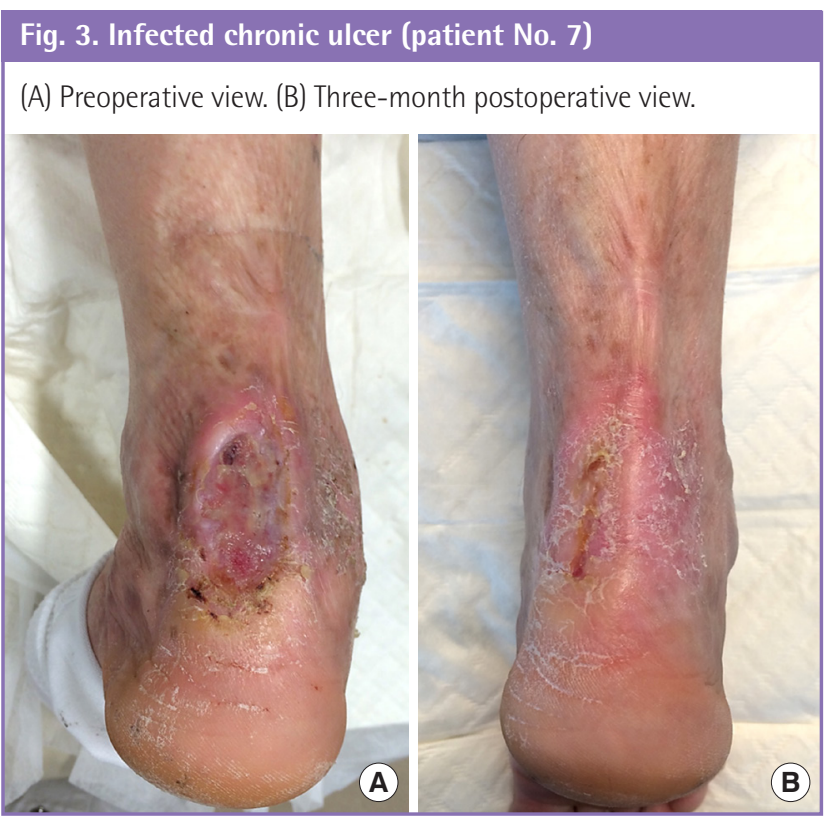

able literature to overcome such limitations and to improve cell content, viability, and take (Table 2 ). The fat was harvested from the lower abdomen, as this has been reported to be the region with the highest ADSC concentration [12,13], although no statistically significant difference in viability has been found compared to other donor sites [14]. Lidocaine has previously been demonstrated to reduce ADSC proliferation and viability, while ropivacaine has been shown to have a negligible effect [15-18]. Epinephrine has not been shown to exhibit any cytotoxic effect [19] and was used in the infiltration solution.

Aspiration was performed according to the micro-fat technique by means of a 3-mm cannula with 1-mm lateral holes. Such an approach has been shown to yield a higher ADSC concentration than a macro-fat approach [20]. Rather than being 
centrifuged, the harvested micro-fat was decanted for $10 \mathrm{~min}$ utes to avoid any possible loss or viability impairment of the ADSCs [21]. The lipoaspirate was then mechanically emulsified by shifting the fat 30 times between two syringes connected by a $90^{\circ}$ Luer-lock connector. No filtration was performed to avoid any loss of ADSCs and to retain the cell fragments resulting from the emulsification process, which may act as pro-inflammatory signals that trigger a regeneration cascade $[4,6]$. The resulting product was injectable with a 27 -gauge needle, allowing for easy tunneling in cases of advanced fibrosis. To accelerate the regenerative process, we combined the fat derivative with PRP $[7,22,23]$. PRP promotes ADSC survival and proliferation both indirectly through neoangiogenesis and directly through the release of factors such as platelet-derived growth factors and transforming growth factor beta [8]. Atashi et al. [23] demonstrated that $20 \%$ non-activated PRP (naPRP) was the most effective concentration in promoting ADSC proliferation, while activated PRP showed inconsistent results. We therefore chose naPRP in our clinical protocol. The naPRP was extracted before starting the surgical procedure to avoid any platelet reduction or activation following the surgical trauma. Specifically, leukocyte-rich PRP (LR-PRP) was chosen, with the expectation that its local antimicrobial activity would support the systemic antibiotic therapy. Such an antibiotic effect was previously demonstrated to be comparable to those of gentamicin and oxacillin against methicillin-sensitive Staphylococcus aureus. In the same study, LR-PRP was shown to inhibit the growth of methicillin-resistant S. aureus and Escherichia coli [22]. The aforementioned modified nanofat graft also plays an antibiotic role. Adipocyte extracts were found to inhibit the growth of $S$. aureus and Pseudomonas aeruginosa by means of the antimicrobial peptide cathelicidin $[24,25]$. Interestingly, in those findings, cathelicidin production initially increased in response to infection, then declined as the adipocytes matured, leading to the deduction that ADSCs or pre-adipocytes were responsible for its production. ADSC-derived cathelicidin and LR-PRP (20\% naLR-PRP) may thus have a synergic antimicrobial effect. Altogether, the reported refinements were made to facilitate improved regenerative and antimicrobial potentials. The technique proved to be effective, as it promoted the healing of infected, surgically-untreatable chronic wounds unresponsive to conventional therapies. In an era of increasing healthcare costs, our approach may reduce the healing time and consequently the patient's distress, the risk of complications, and overall medical expenses. The main weaknesses of this study are the lack of a control group (e.g., PRP alone, nanofat graft alone, negative-pressure wound therapy, etc.) and the small sample size, which hampered subgroup analysis regarding the type of infection and the ulcer etiology. Moreover, different treatments or methods of dressing for 3 months in actual clinical trials may yield similar or better results. Comparison with other therapies was not among the aims of the present study, but it may be an interesting topic for future analyses. In our cohort of patients, we demonstrated that modified nanofat grafting with $20 \%$ naLR-PRP facilitated healing in patients previously unresponsive to antibiotic therapy, surgical debridement, and advanced medications.

\section{NOTES}

\section{Conflict of interest}

No potential conflict of interest relevant to this article was reported.

\section{Ethical approval}

The study was approved by the Institutional Review Board of Policlinico Campus Bio-Medico (IRB No. 3418 PAR) and performed in accordance with the principles of the Declaration of Helsinki. Written informed consents were obtained.

\section{Patient consent}

The patients provided written informed consent for the publication and the use of their images.

\section{Author contribution}

Conceptualization: P Persichetti. Data curation: C Nobile, C Gregorj, V Cerbone, M Gratteri, E Caldaria. Formal analysis: C Nobile, C Gregorj. Methodology: GF Marangi. Project administration: MC Tirindelli, P Persichetti. Visualization: MC Tirindelli. Writing - original draft: M Alessandri-Bonetti, V Cerbone. Writing - review \& editing: F Segreto.

\section{ORCID}

Francesco Segreto https://orcid.org/0000-0002-1573-1098 Giovanni Francesco Marangi

https://orcid.org/0000-0002-7179-3533

Carolina Nobile https://orcid.org/0000-0002-5266-6379 Mario Alessandri-Bonetti

https://orcid.org/0000-0002-5506-8323

Chiara Gregorj https://orcid.org/0000-0003-0789-779X

Vincenzo Cerbone https://orcid.org/0000-0002-1346-0204

Marco Gratteri https://orcid.org/0000-0001-7375-1524

Erika Caldaria https://orcid.org/0000-0003-3882-4171

Maria Cristina Tirindelli

https://orcid.org/0000-0003-4645-4465

Paolo Persichetti https://orcid.org/0000-0001-8857-6060 


\section{REFERENCES}

1. Pourmoussa A, Gardner DJ, Johnson MB, et al. An update and review of cell-based wound dressings and their integration into clinical practice. Ann Transl Med 2016;4:457.

2. Doi K, Ogata F, Eto H, et al. Differential contributions of graft-derived and host-derived cells in tissue regeneration/ remodeling after fat grafting. Plast Reconstr Surg 2015;135: 1607-17.

3. Tabit CJ, Slack GC, Fan K, et al. Fat grafting versus adiposederived stem cell therapy: distinguishing indications, techniques, and outcomes. Aesthetic Plast Surg 2012;36:704-13.

4. Tonnard P, Verpaele A, Peeters G, et al. Nanofat grafting: basic research and clinical applications. Plast Reconstr Surg 2013;132:1017-26.

5. Kemaloglu CA. Nanofat grafting under a split-thickness skin graft for problematic wound management. Springerplus 2016;5:138.

6. Eto H, Kato H, Suga H, et al. The fate of adipocytes after nonvascularized fat grafting: evidence of early death and replacement of adipocytes. Plast Reconstr Surg 2012;129: 1081-92.

7. Cervelli V, Gentile P, Scioli MG, et al. Application of platelet-rich plasma in plastic surgery: clinical and in vitro evaluation. Tissue Eng Part C Methods 2009;15:625-34.

8. D’Esposito V, Passaretti F, Perruolo G, et al. Platelet-rich plasma increases growth and motility of adipose tissue-derived mesenchymal stem cells and controls adipocyte secretory function. J Cell Biochem 2015;116:2408-18.

9. Dohan Ehrenfest DM, Rasmusson L, Albrektsson T. Classification of platelet concentrates: from pure platelet-rich plasma (P-PRP) to leucocyte- and platelet-rich fibrin (LPRF). Trends Biotechnol 2009;27:158-67.

10. Tenna S, Cogliandro A, Barone M, et al. Comparative study using autologous fat grafts plus platelet-rich plasma with or without fractional $\mathrm{CO} 2$ laser resurfacing in treatment of acne scars: analysis of outcomes and satisfaction with FACEQ. Aesthetic Plast Surg 2017;41:661-6.

11. Khouri RK, Smit JM, Cardoso E, et al. Percutaneous aponeurotomy and lipofilling: a regenerative alternative to flap reconstruction? Plast Reconstr Surg 2013;132:1280-90.

12. Padoin AV, Braga-Silva J, Martins P, et al. Sources of processed lipoaspirate cells: influence of donor site on cell concentration. Plast Reconstr Surg 2008; 122:614-8.

13. Jurgens WJ, Oedayrajsingh-Varma MJ, Helder MN, et al. Ef- fect of tissue-harvesting site on yield of stem cells derived from adipose tissue: implications for cell-based therapies. Cell Tissue Res 2008;332:415-26.

14. Rohrich RJ, Sorokin ES, Brown SA. In search of improved fat transfer viability: a quantitative analysis of the role of centrifugation and harvest site. Plast Reconstr Surg 2004;113: 391-7.

15. Keck M, Zeyda M, Gollinger K, et al. Local anesthetics have a major impact on viability of preadipocytes and their differentiation into adipocytes. Plast Reconstr Surg 2010;126: 1500-5.

16. Sung SH, Lee JG, Yu SB, et al. The effects of lidocaine and procaine on microRNA expression of adipocyte-derived adult stem cells. Korean J Anesthesiol 2012;62:552-7.

17. Breu A, Eckl S, Zink W, et al. Cytotoxicity of local anesthetics on human mesenchymal stem cells in vitro. Arthroscopy 2013;29:1676-84.

18. Gugerell A, Kober J, Schmid M, et al. Botulinum toxin A and lidocaine have an impact on adipose-derived stem cells, fibroblasts, and mature adipocytes in vitro. J Plast Reconstr Aesthet Surg 2014;67:1276-81.

19. Girard AC, Atlan M, Bencharif K, et al. New insights into lidocaine and adrenaline effects on human adipose stem cells. Aesthetic Plast Surg 2013;37:144-52.

20. Alharbi Z, Oplander C, Almakadi S, et al. Conventional vs. micro-fat harvesting: how fat harvesting technique affects tissueengineering approaches using adipose tissue-derived stem/ stromal cells. J Plast Reconstr Aesthet Surg 2013;66:1271-8.

21. Conde-Green A, Baptista LS, de Amorin NF, et al. Effects of centrifugation on cell composition and viability of aspirated adipose tissue processed for transplantation. Aesthet Surg J 2010;30:249-55.

22. Kakudo N, Minakata T, Mitsui T, et al. Proliferation-promoting effect of platelet-rich plasma on human adipose-derived stem cells and human dermal fibroblasts. Plast Reconstr Surg 2008;122:1352-60.

23. Atashi F, Jaconi ME, Pittet-Cuenod B, et al. Autologous platelet-rich plasma: a biological supplement to enhance adipose-derived mesenchymal stem cell expansion. Tissue Eng Part C Methods 2015;21:253-62.

24. Zhang LJ, Guerrero-Juarez CF, Hata T, et al. Innate immunity: dermal adipocytes protect against invasive Staphylococcus aureus skin infection. Science 2015;347:67-71.

25. Miller LS. Adipocytes armed against Staphylococcus aureus. N Engl J Med 2015;372:1368-70. 